\title{
Incorporação de lama vermelha na indústria cerâmica: uma revisão
}

\author{
Red mud incorporation in ceramic industry: a review \\ Incorporación de lodo rojo en la industria cerámica: una revisión
}

Recebido: 01/08/2021 | Revisado: 04/07/2021 | Aceito: 07/08/2021 | Publicado: 13/08/2021

Roseane Martins dos Santos

ORCID: https://orcid.org/0000-0002-9162-5182 Instituto Federal de Educação, Ciência e Tecnologia do Piaú, Brasil E-mail: rosyggle@ifpi.edu.br

Rodrigo da Silva Magalhães

ORCID: https://orcid.org/0000-0003-4090-439X Instituto Federal de Educação, Ciência e Tecnologia do Piauí, Brasil E-mail: rmagalhaescaxias@gmail.com

Naiara de Oliveira Sobrinho

ORCID: https://orcid.org/0000-0001-6138-4237 Universidade Federal do Piauí, Brasil E-mail: naiaraoliveira2011@gmail.com

Érico Rodrigues Gomes

ORCID: https://orcid.org/0000-0002-1942-1396 Instituto Federal de Educação, Ciência e Tecnologia do Piaú, Brasil

E-mail: erico.gomes@ifpi.edu.br

\begin{abstract}
Resumo
A lama vermelha é um resíduo proveniente da exploração de bauxita. Nesse contexto, este artigo fornece uma revisão sistemática das pesquisas sobre a adição do resíduo de lama vermelha em tijolos de argila queimados. Composições químicas, materiais e métodos produtivos também são discutidos. São revisadas algumas propriedades dos tijolos como retração linear, massa específica, resistência à compressão e á flexão, porosidade e absorção de água. Além disso são destacadas as propriedades químicas das matérias-primas. Os resultados comuns entre os trabalhos levantados na literatura foram agrupados e discutidos. O reaproveitamento da lama vermelha na produção de tijolos representa uma forma sustentável de promoção de uma economia circular, no qual os resíduos podem ser reutilizados diminuindo a exploração de jazidas de argila. A inserção desse resíduo, em alguns casos, até implica na melhoria das propriedades dos tijolos, bem como uma vantagem produtiva para o setor cerâmico.
\end{abstract}

Palavras-chave: Lama vermelha; Cerâmica; Tijolo de argila.

\begin{abstract}
Red mud is a residue from bauxite mining. A literature review was conducted with applications of this waste in clay bricks. In this context, this paper provides a review of research on the addition of red mud to fired clay bricks. Chemical compositions, materials, and production methods are also discussed. Some brick properties such as linear shrinkage, specific mass, compressive and flexural strength, porosity, and water absorption are reviewed. In addition, the chemical properties of the raw materials are highlighted. The common results among the works surveyed in the literature were grouped and discussed. The reuse of red mud in brick production represents a sustainable way of promoting a circular economy, in which waste can be reused and the exploitation of clay deposits is reduced. The insertion of this waste, in some cases, improves the properties of bricks, as well as representing a productive advantage for the ceramic industry.
\end{abstract}

Keywords: Red mud; Ceramics; Clay brick.

\section{Resumen}

Barro rojo es un residuo proveniente de la explotación de la bauxita. Se realizó una revisión bibliográfica con aplicaciones de estos residuos en ladrillos de arcilla. En este contexto, el presente documento ofrece una revisión de las investigaciones sobre la adición de barro rojo en los ladrillos de arcilla cocida. También se analizan las composiciones químicas, los materiales y los métodos de producción. Se revisan algunas propiedades de los ladrillos, como la contracción lineal, la masa específica, la resistencia a la compresión y a la flexión, la porosidad y la absorción de agua. Además, se destacan las propiedades químicas de las materias primas. Se agruparon y discutieron los resultados comunes entre los trabajos estudiados en la literatura. La reutilización del barro rojo en la producción de ladrillos representa una forma sostenible de promover una economía circular, en la que los residuos pueden reutilizarse reduciendo la explotación de los depósitos de arcilla. La inserción de estos residuos, en algunos casos, mejora las propiedades de los ladrillos, además de representar una ventaja productiva para el sector cerâmico.

Palabras clave: Barro rojo; Cerâmica; Ladrillo de arcilla. 


\section{Introdução}

A lama vermelha (LV) é um resíduo oriundo da produção do alumínio durante o processo Bayer (Kocserha et al., 2018). É uma mistura insolúvel coletada no fundo das autoclaves após a dissolução da bauxita em soda cáustica. É fortemente alcalino $(\mathrm{pH}=13)$ além de conter os seguintes elementos: sílica, alumínio, ferro, cálcio, titânio, sódio, potássio, cromo, vanádio, nióbio, bário, manganês, cobre, chumbo, zinco e outros em concentrações inferiores (Saternus, 2011).

Entre 1 e 1,5 toneladas de lama vermelha são geradas por tonelada de alumina produzida. Estima-se que cerca de 90 milhões de toneladas desse resíduo são gerados todos os anos no mundo (Kocserha et al., 2018; Song et al., 2021). No entanto, a reciclagem segura desta enorme quantidade de resíduos alcalinos é considerada um sério desafio para as indústrias de alumínio devido ao seu pH, conteúdo de elementos radioativos e metais pesados. Logo, a reutilização de LV e sua reinserção na cadeia produtiva é imprescindível tendo em vista esse grande volume gerado (Arroyo et al., 2020).

Atualmente, o descarte da lama vermelha é feito em barragens de contenção projetadas especificamente para essa finalidade. No entanto, essa construção pode representar ameaças ambientais como inundação, penetração subterrânea ou mesmo o rompimento de uma barragem (Babisk et al., 2020). O Brasil é o décimo primeiro país produtor de alumínio primário e o terceiro tanto na produção de alumina quanto na extração de bauxita (Babisk et al., 2020), e diversas pesquisas analisaram a incorporação de LV em materiais cerâmicos (Hildebrando et al., 2013; Macêdo et al., 2011; Ribeiro et al., 2015).

A LV já foi utilizada para produção de agregados leves para construção (Song et al., 2021), geopolímeros (BonetMartínez et al., 2018), remediação do solo (Oprčkal et al., 2020) e adsorção de metais pesados (Mahinroosta et al., 2020). A Figura 1, extraída da base Web of Science, ilustra a quantidade de pesquisas com o termo lama vermelha (red mud).

Figura 1 - $\mathrm{N}^{\circ}$ de publicações acerca da lama vermelha.

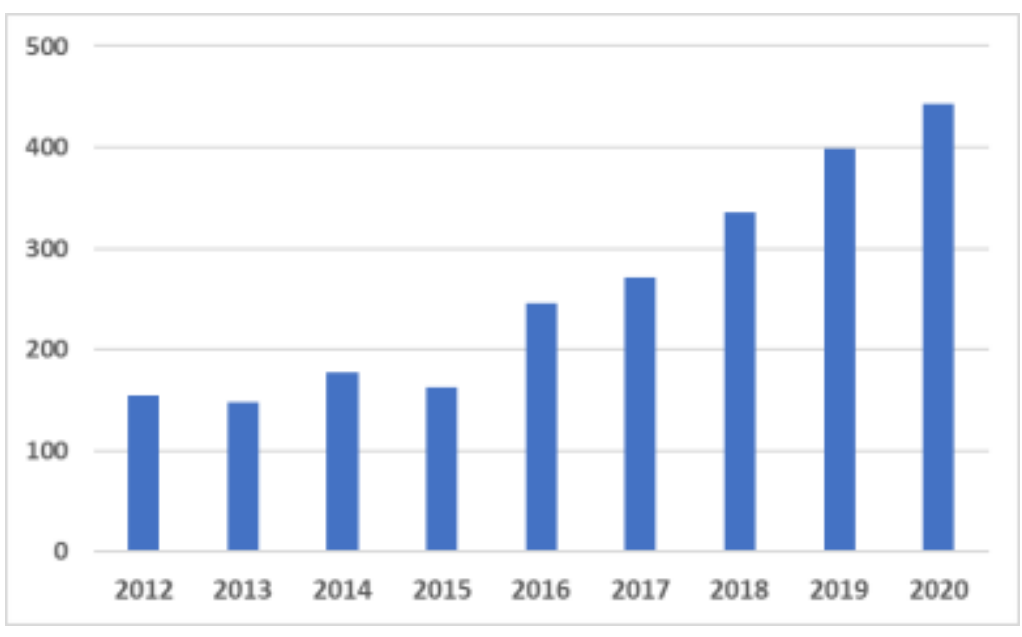

Fonte: Adaptado de Web of Science.

É perceptível, na Figura 1, que a quantidade de pesquisas acerca desse resíduo tem aumentado ano após ano. Portanto, esse dado demonstra a relevância, nessa última década, de pesquisas que tratam de uma destinação alternativa para esse resíduo. Nesse contexto, a incorporação de lama vermelha na indústria da cerâmica vermelha é uma aplicação viável pois esta consome grande volume de matérias-primas (Kocserha et al., 2018). A variabilidade química da matéria-prima argilosa é um fator que contribui para a adição da lama vermelha no processamento cerâmico. Além disso, a LV apresenta um alto teor de ferro e sódio, além de cálcio e outros óxidos fundentes que podem representar vantagens técnicas quanto à sua incorporação em produtos cerâmicos tradicionais, principalmente devido ao processo de sinterização (queima) (Alam et al., 2017; Babisk et al., 2020). Nesse contexto, este artigo tem por objetivo fazer uma revisão sobre a aplicação da lama vermelha na indústria cerâmica. 


\section{Metodologia}

Este trabalho consiste em uma revisão sistemática da literatura científica sobre aplicações do resíduo lama vermelha em tijolos de argila queimados. revisões sistemáticas "identificam um conjunto de estudos já finalizados que abordam uma determinada questão de pesquisa e avaliam os resultados desses estudos para evidenciar conclusões sobre um corpo de conhecimento" (Dermeval et al., 2019, p.3).

A literatura revisada foi selecionada a partir de bases de dados como a ScienceDirect para cobrir as questões discutidas abaixo e compor esta revisão. Os descritores utilizados na busca nas bases de dados foram "red mud", "red mud ceramics" associadas a palavras-chave como "clay" e "clay brick".

Foram incluídos artigos publicados em inglês e português entre 2017-2021. Excluídos artigos duplicados. Selecionou-se artigos que descrevessem pesquisas com aplicações do resíduo de mineração da bauxita, denominado lama vermelha em cerâmica estrutural, tijolos ou blocos de argila queimada.

As questões norteadoras da pesquisa são os principais guias de revisões sistemáticas da literatura, uma vez que norteiam as atividades da pesquisa e são imprescindíveis para o sucesso de sua execução (Dermeval et al., 2019). Portanto, conforme finalizado o processo de seleção dos artigos, as questões norteadoras seguiram as seguintes considerações: Quais são os materiais? Quais são os métodos utilizados? Quais são as propriedades do resíduo tratado? Quais as proporções para substituição de argila? Qual é o significado e a importância do aspecto de produção mais limpa e preocupações ambientais e sustentáveis no artigo? Quais são as principais conclusões e perspectivas dos artigos?

\section{Resultados}

A Figura 2 apresenta o resultado das buscas realizadas nas bases de dados. Após aplicada a estratégia de busca, o levantamento inicial resultou em 18 artigos na ScienceDirect e 85 na Web of Science. No entanto, após a aplicação dos critérios de inclusão, selecionou-se 11 artigos, dos quais 4 apareciam duplicados. Logo, no recorte final, foram revisados 7 artigos.

Figura 2. Encadeamento da pesquisa.

\begin{tabular}{|c|c|}
\hline \multicolumn{2}{|c|}{ PERÍODO/IDIOMA } \\
\hline $2017-2021$ & Português/inglês \\
\hline \multicolumn{2}{|c|}{ BASE DE DADOS } \\
\hline ScienceDirect & Web of Science \\
\hline \multicolumn{2}{|c|}{ ESTRATÉGIA DE BUSCA } \\
\hline \multicolumn{2}{|c|}{$\begin{array}{c}\text { ("red mud" OR "red mud ceramics") } \\
\text { AND "clay" }\end{array}$} \\
\hline \multicolumn{2}{|c|}{ RESULTADOS } \\
\hline 18 & 85 \\
\hline \multicolumn{2}{|c|}{ SELECIONADOS/DUPLICADOS } \\
\hline 11 & 4 \\
\hline
\end{tabular}

Fonte: Autores. 


\section{Discussões}

\subsection{Propriedades físico-químicas}

O Quadro 1 compila a composição química da lama vermelha (LV) caracterizada por vários autores. Este resíduo industrial consiste em uma mistura de óxidos $\mathrm{Fe}_{2} \mathrm{O}_{3}, \mathrm{Al}_{2} \mathrm{O}_{3}, \mathrm{SiO}_{2}, \mathrm{TiO}_{2}$ e $\mathrm{Na}_{2} \mathrm{O}$ como teores principais, mas também $\mathrm{CaO}$ em quantidade considerável. No entanto, a quantidade de cada óxido depende da bauxita original utilizada no processo Bayer e de como o processo foi realizado, e portanto, varia bastante conforme a técnica industrial empregada (Babisk et al., 2020; Silva Filho et al., 2007). É nítido que, independentemente do autor, a quantidade de óxido de ferro foi preponderante e, além disso, a presença desse elemento confere a cor avermelhada característica da LV (Mahinroosta et al., 2020).

Quadro 1 - Composição química da LV.

\begin{tabular}{|c|c|c|c|c|c|}
\hline Autor & $\begin{array}{c}\text { (Arroyo et al., } \\
2020)\end{array}$ & $\begin{array}{c}\text { (Babisk et al., } \\
2020)\end{array}$ & $\begin{array}{c}\text { (Xu et al., } \\
2019)\end{array}$ & $\begin{array}{c}\text { (Scribot et al., } \\
2018)\end{array}$ & $\begin{array}{c}\text { (Alekseev et } \\
\text { al., 2019) }\end{array}$ \\
\hline $\mathrm{SiO}_{2}$ & 4,87 & 12,2 & 13,55 & 5,9 & 15,5 \\
\hline $\mathrm{Al}_{2} \mathrm{O}_{3}$ & 18,08 & 22,11 & 18,96 & 12,8 & 21,2 \\
\hline $\mathrm{Fe}_{2} \mathrm{O}_{3}$ & 50,89 & 34,49 & 30,16 & 59,1 & 29,9 \\
\hline $\mathrm{CaO}$ & 1,13 & 3,84 & 8,73 & 5,4 & 4,2 \\
\hline $\mathrm{K} 2 \mathrm{O}$ & 0,07 & 0,66 & 0,11 & - & 0,4 \\
\hline $\mathrm{MgO}$ & 0,07 & - & 0,14 & 0,1 & - \\
\hline $\mathrm{Na}_{2} \mathrm{O}$ & 3,45 & 5,31 & 8,48 & 3,8 & 10,3 \\
\hline $\mathrm{TiO}_{2}$ & 9,33 & 3,61 & 6,96 & 10,4 & 2,4 \\
\hline $\mathrm{P}_{2} \mathrm{O}_{5}$ & 0,45 & 0,45 & - & 0,6 & 0,6 \\
\hline $\mathrm{MnO}_{2}$ & 0,07 & - & - & - & 0,2 \\
\hline $\mathrm{P.F}$ & 10,26 & 16,1 & 12,91 & 7,47 & 14,4 \\
\hline
\end{tabular}

Fonte: Autores.

A lama vermelha contém uma grande quantidade de $\mathrm{SiO}_{2}, \mathrm{Al}_{2} \mathrm{O}_{3}, \mathrm{CaO}$ etc., todos os principais componentes do vidro e da cerâmica, portanto, esse resíduo tem potencial na produção desses materiais (Wang et al., 2021). Em geral, a LV se apresenta como um material fino, com tamanho de sua partícula na faixa de 0,1 a $160 \mu \mathrm{m}$. A área específica varia entre 10 e 31 $\mathrm{m}^{2} / \mathrm{g}$, o que indica que a LV possui um alto grau de dispersão de partículas minerais (Sahu \& Patel, 2016).

\subsection{Incorporação da lama vermelha na cerâmica}

A produção de produtos cerâmicos e materiais de construção contendo diferentes quantidades de lama vermelha é uma alternativa viável para diminuir esse passivo ambiental. A utilização de LV como matéria-prima secundária para a produção de tijolos pode atingir o aproveitamento abrangente de quase 100\% do resíduo produzido (Arroyo et al., 2020). Além disso, existem muitas substâncias na LV que podem diminuir a temperatura de sinterização para economizar energia e ajudar a formar uma fase vítrea para melhorar a resistência dos tijolos (He et al., 2012). O Quadro 2 compila as principais metodologias utilizadas no processamento desses materiais nos artigos revisados. 
Quadro 2 - Processo produtivo.

\begin{tabular}{|c|c|c|c|}
\hline Autor & Composição & Processo produtivo & Moldagem/Corpos de prova \\
\hline $\begin{array}{c}\text { (Scribot et al., } \\
\text { 2018) }\end{array}$ & $0,10,20$ e $30 \%$ & $\begin{array}{c}\text { Secagem e moagem, e queima a } 950 \text { e } \\
1015^{\circ} \mathrm{C} \text { por } 2 \text { horas (patamar) }\end{array}$ & $\begin{array}{l}\text { Por extrusão/ Dimensões 4x4x16 cm e } \\
\qquad 15 \times 11 \times 1,5\end{array}$ \\
\hline $\begin{array}{l}\text { (Kocserha et al., } \\
\text { 2018) }\end{array}$ & 0 a $50 \%$ & $\begin{array}{c}\text { Secagem por } 24 \text { horas em } \\
\text { temperatura ambiente e por } 48 \text { horas } \\
\text { a } 90^{\circ} \mathrm{C} \text {, e queima a } 860^{\circ} \mathrm{C} \\
\end{array}$ & Por extrusão/Dimensões 2,4x5x15 cm \\
\hline $\begin{array}{l}\text { (Alekseev et al., } \\
\text { 2019) }\end{array}$ & 50 a $100 \%$ & $\begin{array}{c}\text { Secagem a } 100^{\circ} \mathrm{C} \text {, e queima } 800,900, \\
1000,1050,1100,1150,1200 \text { e } 1225 \\
{ }^{\circ} \mathrm{C} \text { por } 3 \text { horas (patamar) }\end{array}$ & $\begin{array}{l}\text { Por prensagem }(5 \mathrm{MPa}) / \text { Dimensões } \\
\qquad 6 \times 2 \times 1 \mathrm{~cm}\end{array}$ \\
\hline $\begin{array}{l}\text { (Hamza et al., } \\
\text { 2019) }\end{array}$ & 0 a $30 \%$ & - & $\begin{array}{l}\text { Por prensagem } / 3,3 \mathrm{~cm} \text { de diâmetro e } 4 \\
\text { de comprimento }\end{array}$ \\
\hline (Xu et al., 2019) & 0 e $40 \%$ & $\begin{array}{c}\text { Mistura após Secagem a } 110^{\circ} \mathrm{C} \text { por } \\
24 \mathrm{~h} \text {, e queima de } 940 \text { a } 1160^{\circ} \mathrm{C} \text { por } 2 \\
\text { horas (patamar) }\end{array}$ & $\begin{array}{l}\text { Por prensagem (40MPa) Dimensões } \\
\qquad 3,7 \times 0,65 \times 0,65 \mathrm{~cm}\end{array}$ \\
\hline $\begin{array}{l}\text { (Arroyo et al., } \\
\text { 2020) }\end{array}$ & 0,50 e $80 \%$ & $\begin{array}{c}\text { Secagem a } 105^{\circ} \mathrm{C} \text { por } 48 \mathrm{~h} \text {, e queima } \\
\text { a } 900 \text { e } 1100{ }^{\circ} \mathrm{C}\end{array}$ & $\begin{array}{l}\text { Por prensagem }(50 \mathrm{MPa}) \text { Corpo de } \\
\text { prova cilíndrico } 3,3 \mathrm{~cm} \text { de diâmetro por } \\
4 \mathrm{~cm} \text { de comprimento }\end{array}$ \\
\hline $\begin{array}{l}\text { (Babisk et al., } \\
\text { 2020) }\end{array}$ & 0 a $100 \%$ & $\begin{array}{c}\text { Secagem a } 45^{\circ} \mathrm{C} \text { e } 105^{\circ} \mathrm{C} \text { por } 24 \mathrm{~h} \text {, e } \\
\text { queima a } 850,950 \text { e } 1050^{\circ} \mathrm{C} \text { por } 2 \\
\text { horas (patamar de queima) }\end{array}$ & $\begin{array}{l}\text { Por prensagem (20 MPa) Dimensões } \\
\qquad 12 \times 3 \times 1,2 \mathrm{~cm}\end{array}$ \\
\hline
\end{tabular}

Fonte: Autores.

Em geral, o método de prensagem possibilita a incorporação de uma quantidade maior de resíduo. Isto se dá, porque conformar os corpos de prova por extrusão requer uma plasticidade mínima, e quanto mais resíduo é incorporado, menor a plasticidade da massa (Hamza et al., 2019). As temperaturas de queima variaram de 850 até $1.225^{\circ} \mathrm{C}$. Além disso, os dados do Quadro 2 demonstram pesquisas que incorporam até $100 \%$ de LV, ou seja, substituindo completamente a matéria-prima argilosa nos tijolos cerâmicos.

O Quadro 3 compila dados das propriedades tecnológicas de vários autores tais como retração linear de secagem (RLs), retração linear de queima (RLq), absorção de água (AA), massa específica aparente (MEA), porosidade aparente (PA), resistência à compressão (RC) e tensão de ruptura à flexão (TRF). Percebe-se que a maioria dos valores de AA variaram abaixo dos limites máximos de $22 \%$ para a fabricação de tijolos (ABNT, 2017). Além disso, outro parâmetro importante em que se observaram excelentes resultados é a resistência mecânica, a qual tem uma variação aceitável entre 4 e 30 Mpa (Dondi, 2006). Nota-se valores para RLq em Kocserha et al. (2018) bem acima dos valores padrões (<3\%) (Dondi, 2006). O autor ressalta que as matérias-primas argilosas contém alto índice de carbonatos e que a LV não influenciou nos resultados da RLq.

Quadro 3 - Resumo das propriedades tecnológicas com adição de LV.

\begin{tabular}{|c|c|c|c|c|c|c|}
\hline Autor & RLs (\%) & $\begin{array}{c}\text { RLq } \\
(\%)\end{array}$ & AA $(\%)$ & $\begin{array}{c}\text { MEA } \\
\left(\mathbf{g} / \mathbf{c m}^{3}\right)\end{array}$ & PA (\%) & RC ou TRF (Mpa) \\
\hline (Scribot et al., 2018) & $3,8-4,7$ & $0,1-2$ & $10,8-15,7$ & $\begin{array}{c}1,87- \\
1,91\end{array}$ & $31-37$ & $\begin{array}{c}\text { RC de } 38,8-73,2 \\
\text { TRF de } 15,3-19,1\end{array}$ \\
\hline $\begin{array}{c}\text { (Kocserha et al., } \\
\text { 2018) }\end{array}$ & $12,2-20,4$ & $\begin{array}{c}18,3- \\
31,2\end{array}$ & $14,2-22,5$ & $\begin{array}{c}1,81- \\
2,04\end{array}$ & - & $\begin{array}{c}\text { RC de 23,8-42,1 } \\
\text { TRF de } 9,12-15,18\end{array}$ \\
\hline $\begin{array}{c}\text { (Alekseev et al., } \\
\text { 2019) }\end{array}$ & - & $\begin{array}{c}1,71- \\
12,45\end{array}$ & $\begin{array}{c}2,35- \\
33,78\end{array}$ & $\begin{array}{c}1,28- \\
2,11\end{array}$ & - & TRF de 0-12,32 \\
\hline (Xu et al., 2019) & - & - & $1-19$ & $1,5-2,7$ & $1-35$ & TRF de $18-63$ \\
\hline (Arroyo et al., 2020) & - & - & $8-35$ & $1,5-2,1$ & - & RC de 1-17 \\
\hline (Babisk et al., 2020) & - & $0,25-6$ & $12,5-32,4$ & $\begin{array}{c}0,53- \\
0,88\end{array}$ & - & RC de $1-13$ \\
\hline
\end{tabular}

Fonte: Autores. 
Kocserha et al. (2018) após a extrusão, secaram as amostras por 24 horas em temperatura ambiente e por 48 horas em câmara de secagem a $90^{\circ} \mathrm{C}$. Após esse processo, os autores mensuraram o pH dos corpos de prova. Embora a LV seja altamente alcalina $(\mathrm{pH}=13)$, constatou-se que o $\mathrm{pH}$ dos tijolos cerâmicos com incorporação da LV se mostrou neutro viabilizando sua aplicação industrial. Além disso, realizaram um estudo analisando duas argilas, uma com alto teor de carbonatos (A1), e outra sem (A2). Tijolos incorporados com lama vermelha (0 a 50\% em substituição) produzidos com ambas as argilas e queimados a $860^{\circ} \mathrm{C}$.

Os resultados demonstraram influência do teor de carbonatos na retração linear de queima que se manteve sempre maior nos compostos A1 e adição de lama vermelha (LV) do que em A2. Em todos os casos, as amostras de A1 apresentaram menor densidade devido ao alto teor de carbonato. Além disso, O teor de até $30 \%$ de LV, quer seja com A1 ou A2 até $30 \%$ não teve alteração considerável na absorção de água. Com a $\mathrm{A} 1$, a resistência à compressão com até $40 \%$ de $\mathrm{LV}$ aumentou constantemente de 23,8 para 36,8 Mpa. Portanto, Kocserha et al. (2018) ressaltam que a incorporação de LV para fabricação de tijolos de argila obteve resultados satisfatórios.

Arroyo et al. (2020) realizaram um estudo incorporando LV nas proporções 0, 50 e $80 \%$ e temperaturas de queima em 900 e $1100^{\circ} \mathrm{C}$. O teste de lixiviação demonstra que altas concentrações de metais como Se, Cr e Zn ultrapassaram os limites recomendados. Os ensaios tecnológicos demonstraram que, à medida que se adiciona $\mathrm{LV}$, na temperatura de $1100^{\circ} \mathrm{C}$, o tijolo aumentou a densidade, diminuiu e incrementou a resistência à compressão. Na temperatura de $900^{\circ} \mathrm{C}$, quanto maior a incorporação de LV menor foi a densidade e resistência à compressão.

Babisk et al. (2020) analisaram a incorporação de LV para fabricação de materiais para construção civil (tijolos e telhas). Sete formulações (C1 a C7) foram queimadas a 850,950 e $1050^{\circ} \mathrm{C}$ a uma taxa de $2^{\circ} \mathrm{C} / \mathrm{min}$. A C7 (0\% de resíduo) obteve maior massa específica, o que refletiu na menor taxa de absorção de água. Os resultados do ensaio de resistência à compressão sugeriram a adição de LV somente em altas temperaturas $\left(>1050^{\circ} \mathrm{C}\right)$. $\mathrm{O}$ ensaio de lixiviação demonstra que $\mathrm{LV}$ investigada é classificada como classe II, resíduo não perigoso, de acordo com os padrões brasileiros, mas não foi considerada tóxica.

Xu et al., (2019) analisaram a adição de quatro tipos de lamas vermelhas (A1 a A4) em formulações para revestimento cerâmico. As 4 formulações foram submetidas a temperaturas que variaram entre 940 e $1160^{\circ} \mathrm{C}$. Os melhores resultados advêm das amostras A2 (40\% de LV) e A3 (40\% de LV) das lamas vermelhas, uma vez que, nas temperaturas de $1100-1140^{\circ} \mathrm{C}$, obtiveram resultados absorção de água inferior a $2 \%$ e tensões de ruptura à flexão superiores a 50Mpa. Portanto, ambas as formulações satisfizeram todas as recomendações normativas.

Scribot et al. (2018) estudaram a adição de uma lama vermelha modificada (LVm) na produção de tijolos e ladrilhos cerâmicos. O resíduo é decorrente de um processo inovador que extrai o hidróxido de sódio usado no processo de beneficiamento da alumina. Após esse processo, o resíduo foi adicionado nas proporções (10, 20 e 30\%) e queimado em duas temperaturas $\left(950\right.$ e $\left.1015^{\circ} \mathrm{C}\right)$. Pode-se notar que a formulação contendo $30 \%$ de $\mathrm{LVm}$, na temperatura de $1015^{\circ} \mathrm{C}$ exibe resistências à compressão substancialmente maiores (64,9 MPa) do que as resistências à compressão de misturas contendo 10 e $20 \%$ de lama vermelha modificada (54,2 e 39,1 MPa respectivamente). Em relação aos aspectos ambientais, as concentrações de metais pesados estão abaixo dos limites regulamentares para resíduos perigosos exceto para as amostras contendo $30 \%$ de LV e sinterizadas a $950^{\circ} \mathrm{C}$.

Alekseev et al. (2019) analisaram as propriedades de tijolos confeccionados por prensagem com adições de dois resíduos: a lama vermelha (50 a 100\%) e areia de fundição (0 a 50\%). As temperaturas de queima foram 800, 900, 1000, 1050, $1100,1150,1200$ e $1225^{\circ} \mathrm{C}$ por 3 horas (patamar). Os resultados demonstram que os corpos de prova compostos com $100 \%$ de lama vermelha obtiveram resistência à flexão de 12,17 e $12,32 \mathrm{Mpa}$, a 1200 e $1225^{\circ} \mathrm{C}$, respectivamente. As formulações atenderam ao limite máximo de $20 \%$ para absorção de água somente a partir da temperatura de $1150^{\circ} \mathrm{C}$, no entanto, a retração 
linear aumentou com o incremento da temperatura, de $1,89 \%\left(800^{\circ} \mathrm{C}\right)$ até $12,45 \%\left(1225^{\circ} \mathrm{C}\right)$.

Hamza et al. (2019) analisaram a influência da lama vermelha na plasticidade da massa cerâmica. Dois métodos foram utilizados para mensurar a plasticidade: método de Pferfferkorn e teste de compressão. Os resultados, em ambos os métodos, apontam para diminuição da plasticidade das massas com adição de lama vermelha. Portanto, o autor conclui ressaltando a viabilidade da utilização do resíduo em até $30 \%$ sem depreender energia e recursos para beneficiá-lo através de processos de moagem ou secagem.

Kocserha et al. (2018) relatam alguns entraves à incorporação desse resíduo na indústria cerâmica. Além da secagem, a moagem da lama vermelha demandaria grande consumo de energia e a introdução da lama vermelha no processamento cerâmico também requer a construção de uma linha tecnológica para preparação de matérias-primas, ou seja, secadores especiais e moinhos com sistema de tecnologia de pó.

\section{Considerações Finais}

Este trabalho investigou a aplicação da lama vermelha (LV), um subproduto da exploração da bauxita, na cerâmica vermelha. Sete artigos foram encontrados na literatura. Os resultados mostraram que é totalmente viável a incorporação desse resíduo no processamento cerâmico e, em alguns casos, até melhorou as propriedades tecnológicas dos tijolos. Além disso, essa destinação promove a inertização desse subproduto que é rico em metais pesados. Portanto, a aplicação de LV na indústria cerâmica se constitui numa alternativa sustentável para redução desse passivo ambiental.

A enorme quantidade de lama vermelha descarregada por indústrias produtoras de alumina a partir de bauxita representa um problema ambiental e econômico, portanto, novas pesquisas voltadas para estudos das aplicações tecnológicas deste resíduo devem ser incentivadas. O aumento do número de pesquisas na última década reforça essa demanda, principalmente no desenvolvimento de novos produtos cerâmicos (tijolos, telhas, porcelanatos, entre outros) ou simples adição de lama vermelha no processo produtivo destes, visto que a indústria cerâmica possui alto potencial de absorção de resíduos em sua cadeia produtiva.

\section{Referências}

ABNT. (2017). Componentes cerâmicos - Blocos e tijolos para alvenaria. Associação Brasileira de Normas Técnicas.

Alam, S., Das, S. K., \& Rao, B. H. (2017). Characterization of coarse fraction of red mud as a civil engineering construction material. Journal of Cleaner Production, 168, 679-691. https://doi.org/10.1016/j.jclepro.2017.08.210.

Alekseev, K., Mymrin, V., Avanci, M. A., Klitzke, W., Magalhães, W. L. E., Silva, P. R., Catai, R. E., Silva, D. A., \& Ferraz, F. A. (2019). Environmentally clean construction materials from hazardous bauxite waste red mud and spent foundry sand. Construction and Building Materials, 229. https://doi.org/10.1016/j.conbuildmat.2019.116860.

Arroyo, F., Luna-Galiano, Y., Leiva, C., Vilches, L. F., \& Fernández-Pereira, C. (2020). Environmental risks and mechanical evaluation of recycling red mud in bricks. Environmental Research, 186(April), 109537. https://doi.org/10.1016/j.envres.2020.109537.

Babisk, M. P., Amaral, L. F., Ribeiro, L. D. S., Vieira, C. M. F., Prado, U. S. Do, Gadioli, M. C. B., Oliveira, M. S., Luz, F. S. Da, Monteiro, S. N., \& Garcia Filho, F. D. C. (2020). Evaluation and application of sintered red mud and its incorporated clay ceramics as materials for building construction. Journal of Materials Research and Technology, 9(2), 2186-2195. https://doi.org/10.1016/j.jmrt.2019.12.049.

Bonet-Martínez, E., Pérez-Villarejo, L., Eliche-Quesada, D., Carrasco-Hurtado, B., Bueno-Rodríguez, S., \& Castro-Galiano, E. (2018). Inorganic polymers synthesized using biomass ashes-red mud as precursors based on clay-kaolinite system. Materials Letters, $225,161-166$. https://doi.org/10.1016/j.matlet.2018.05.012.

Dermeval, D., Coelho, J. A. P. de M., \& Bittencourt, I. I. (2019). Mapeamento Sistemático e Revisão Sistemática da Literatura em Informática na Educação. In Metodologia de Pesquisa em Informática na Educação: Abordagem Quantitativa de Pesquisa. SBC. https://metodologia.ceie-br.org/livro-2/

Dondi, M. (2006). Caracterização Tecnológica dos Materiais Argilosos: Métodos Experimentais e Interpretação dos Dados. Cerâmica Industrial, 11(3), 36-40.

Hamza, A., Kocserha, I., Géber, R., \& Buzimov, A. (2019). Plasticity of Red Mud and Clay Mixtures. IOP Conference Series: Materials Science and Engineering, 613(1). https://doi.org/10.1088/1757-899X/613/1/012051. 
Research, Society and Development, v. 10, n. 10, e321101018949, 2021 (CC BY 4.0) | ISSN 2525-3409 | DOI: http://dx.doi.org/10.33448/rsd-v10i10.18949

He, H., Yue, Q., Qi, Y., Gao, B., Zhao, Y., Yu, H., Li, J., Li, Q., \& Wang, Y. (2012). The effect of incorporation of red mud on the properties of clay ceramic bodies. Applied Clay Science, 70, 67-73. https://doi.org/10.1016/j.clay.2012.09.022.

Hildebrando, E. A., Da Silva Souza, J. A., Angélica, R. S., \& De Freitas Neves, R. (2013). Application of bauxite waste from amazon region in the heavy clay industry. Materials Research, 16(6), 1418-1422. https://doi.org/10.1590/S1516-14392013005000145.

Kocserha, I., Hamza, A., \& Géber, R. (2018). The effects of red mud on clay compounds. IOP Conference Series: Materials Science and Engineering, 426(1). https://doi.org/10.1088/1757-899X/426/1/012026.

Macêdo, A. N., Costa, D. H. P. e, Trindade, S. R. dos S., Souza, J. A. da S., \& Carneiro, R. J. de F. M. (2011). Comportamento de blocos cerâmicos estruturais produzidos a partir da mistura de lama vermelha e argila. Ambiente Construído, 11(4), 25-36. https://doi.org/10.1590/s1678-86212011000400003.

Mahinroosta, M., Karimi, Z., \& Allahverdi, A. (2020). Recycling of Red Mud for Value-Added Applications: A Comprehensive Review. In Encyclopedia of Renewable and Sustainable Materials. Elsevier Ltd. https://doi.org/10.1016/b978-0-12-803581-8.11474-2.

Oprčkal, P., Mladenovič, A., Zupančič, N., Ščančar, J., Milačič, R., \& Zalar Serjun, V. (2020). Remediation of contaminated soil by red mud and paper ash. Journal of Cleaner Production, 256. https://doi.org/10.1016/j.jclepro.2020.120440.

Ribeiro, L. da S., Babisk, M. P., Do Prado, U. S., Monteiro, S. N., \& Vieira, C. M. F. (2015). Incorporation of in natura and calcined red muds into clay ceramic. Materials Research, 18(Suppl 2), 279-282. https://doi.org/10.1590/1516-1439.372014.

Sahu, M. K., \& Patel, R. K. (2016). Methods for Utilization of Red Mud and Its Management. In Environmental Materials and Waste: Resource Recovery and Pollution Prevention. Elsevier Inc. https://doi.org/10.1016/B978-0-12-803837-6.00019-6.

Saternus, M. (2011). Bayern's method of Al2O3Production - Possibilities of red mud disposal and utilization. Solid State Phenomena, 176, 11-20. https://doi.org/10.4028/www.scientific.net/SSP.176.11.

Scribot, C., Maherzi, W., Benzerzour, M., Mamindy-pajany, Y., \& Abriak, N. (2018). A laboratory-scale experimental investigation on the reuse of a modified red mud in ceramic materials production. 163, 21-31.

Silva Filho, E. B., Alves, M. C. M., \& Da Motta, M. (2007). Lama vermelha da indústria de beneficiamento de alumina: produção, características, disposição e aplicações alternativas. Matéria (Rio de Janeiro), 12(2), 322-338. https://doi.org/10.1590/s1517-70762007000200011.

Song, C., Zhang, H., Dong, Y., Pei, L., Liu, H., Jiang, J., \& Xu, H. (2021). Investigation on the fabrication of lightweight aggregate with acid-leaching tailings of vanadium-bearing stone coal minerals and red mud. Chinese Journal of Chemical Engineering. https://doi.org/10.1016/j.cjche.2020.09.020.

Wang, S., Jin, H., Deng, Y., \& Xiao, Y. (2021). Comprehensive utilization status of red mud in China: A critical review. Journal of Cleaner Production, 289. https://doi.org/10.1016/j.jclepro.2020.125136.

Xu, X., Song, J., Li, Y., Wu, J., Liu, X., \& Zhang, C. (2019). The microstructure and properties of ceramic tiles from solid wastes of Bayer red muds. Construction and Building Materials, 212, 266-274. https://doi.org/10.1016/j.conbuildmat.2019.03.280 